\title{
Application Of Inclusion Measure In Interval Valued Neutrosophic Sets
}

\author{
S. Ramya ${ }^{1}$, N. Sathya Seelan ${ }^{2}$ \& K. Balasangu ${ }^{3}$ \\ ${ }^{1}$ Research Scholar, Thiru Kolanjiappar Government Arts College (Grade-1), \\ Vriddhachalam - 606 001, Tamilnadu. \\ ${ }^{2,3}$ Assistant Professor in Mathematics, Thiru Kolanjiappar Government Arts College (Grade-1), \\ Vriddhachalam - 606 001, Tamilnadu.
}

\begin{abstract}
In this paper, we discuss about Inclusion Measure for ranking the Interval Valued Neutrosophic Sets (IVNSs) by using some distance measure concepts. Inclusion Measure is an easy way to understand the ranking method between IVNSs. We finally illustrate a multi attribute decision making problem to reveal the strength and usefulness of Inclusion Measure.
\end{abstract}

\section{Key Words}

Interval Valued Neutrosophic Sets, Distance Measure, Inclusion Measure, Multi attribute decision making.

\section{Introduction}

L. A. Zadeh introduced the Fuzzy Set (FS) theory in 1965 which handle Uncertainty. A FS is a set having each element with membership grade within [0,1]. The generalization of Fuzzy Sets, called Intuitionistic Fuzzy Set (IFS), is introduced by K. Atanassov in 1986. An IFS is a set with each member having membership and non-membership grades within [0,1]. In 1995, Florentin Smarandache introduced Neutrosophic Set (NS) which is the generalization of FS and IFS. In NS, each element has truth membership, indeterminacy membership and falsity membership which are independent and within [0,1]. Further, in 2005, Wang et. al., developed an occurrence of NS called Single Valued Neutrosophic Sets (SVNSs). Later they also have introduced Interval Valued Neutrosophic Sets (IVNSs) which expressed in closed subintervals of [0,1]. Şahin and Küçük proposed the subsethood (inclusion) measure for SVNSs and applied it to a multi-criteria decision making problem recently. We extend the Inclusion Measure of SVNSs to IVNSs in this paper. And, we use the Neutrosophic Inclusion Measure to rank the IVNSs by considering a multi attribute decision making problem to indicate the potential of the proposed inclusion measure. 


\section{Preliminaries}

\section{Definition - Neutrosophic Set}

Let $X$ be a Universal set with a generic element $x \in X$. A Neutrosophic Set (NS) $R$ in $X$ is defined as $R=\left\{\left\langle x, A_{R}(x)\right\rangle: x \in X\right\}$ where $A_{R}(x)$ is the ordered triplet $\left(T_{R}(x), I_{R}(x), F_{R}(x)\right)$ of three numbers corresponding to each $x . T_{R}(x), I_{R}(x)$ and $F_{R}(x)$ are real standard or non-standard subsets of ]$^{-} 0,1^{+}\left[\text {. That is, Each of } T_{R}, I_{R}, F_{R}: X \rightarrow\right]^{-} 0,1^{+}[$. There is no restriction on the sum $T_{R}(x), I_{R}(x)$ and $F_{R}(x)$, so ${ }^{-} 0 \leq \sup T_{R}(x)+\sup I_{R}(x)+\sup F_{R}(x) \leq 3^{+}$.

Here, $T_{R}(x), I_{R}(x)$ and $\mathrm{F}_{R}(x)$ are the truth membership, indeterminacy membership and falsity membership degrees of the element $x$ to the set $R$. Note that NS represented in the philosophical point of view earlier. But, through scientific point of view, Wang improved $\left({ }^{-} 0,1^{+}\right)$to $[0,1]$ for reality.

\section{Definition - Single Valued Neutrosophic Set}

Let $X$ be a Universal set. A Single Valued Neutrosophic Set (SVNS) $R$ over $X$ is an object having the form $R=\left\{\left\langle x, T_{R}(x), I_{R}(x), F_{R}(x)\right\rangle: x \in X\right\}$, where $T_{R}(x), I_{R}(x), F_{R}(x): X \rightarrow[0,1]$ with $0 \leq T_{R}(x)+I_{R}(x)+F_{R}(x) \leq 3$ for all $x \in X$. Here, $T_{R}(x), I_{R}(x)$ and $\mathrm{F}_{R}(x)$ are the truth membership, indeterminacy membership and falsity membership degrees of the element $x$ to the set $R$ respectively.

\section{Definition - Interval Valued Neutrosophic Set}

Let $X$ be a Universal set. An Interval Valued Neutrosophic Set (IVNS) is defined with the form $R=\left\{\left\langle x, T_{R}(x), I_{R}(x), F_{R}(x)\right\rangle: x \in X\right\}$, where $T_{R}(x), I_{R}(x), F_{R}(x): X \rightarrow$ int $[0,1]$ with $0 \leq \sup T_{R}(x)+\sup I_{R}(x)+\sup F_{R}(x) \leq 3$ for all $x \in X$. That is,

$$
R=\left\{\left\langle x,\left[T_{R}^{l}(x), T_{R}^{u}(x)\right],\left[I_{R}^{l}(x), I_{R}^{u}(x)\right],\left[F_{R}^{l}(x), F_{R}^{u}(x)\right]\right\rangle: x \in X\right\}
$$

Note that, $T_{R}(x)=\left[T_{R}^{l}(x), T_{R}^{u}(x)\right] ; \quad I_{R}(x)=\left[I_{R}^{l}(x), I_{R}^{u}(x)\right]$ and $F_{R}(x)=\left[F_{R}^{l}(x), F_{R}^{u}(x)\right]$. 


\section{Definition - Inclusion Measure}

Let $X$ be a set containing IVNSs. A mapping $I: X \times X \rightarrow[0,1]$ is said to be an inclusion measure for IVNSs if $I$ satisfies the following properties: For all $P, Q, R \in X$,

(1) $I(P, Q)=1$ if $P \subseteq Q$

(2) $I\left(P, P^{c}\right)=1$ iff $\left[T_{P}^{l}(x), T_{P}^{u}(x)\right] \leq\left[I_{P}^{l}(x), I_{P}^{u}(x)\right]$ and $\left[F_{P}^{l}(x), F_{P}^{u}(x)\right] \leq[0.5,0.5]$ for all $x \in X$

(3) $I\left(\underline{-}_{-}, 0\right)=0$, where 1 is the interval absolute NS and ${ }_{-}^{0}$ is the interval empty NS.

(4) $P \subseteq Q \subseteq R \Rightarrow I(R, P) \leq I(Q, P)$ and $I(R, P) \leq I(R, Q)$

Note

$I(A, B)$ represents an inclusion measure expressing the degree to which $A$ belongs to $B$.

\section{Application of Interval Valued Neutrosophic Fuzzy Sets in Pattern Recognition}

Now we illustrate a multi-attribute decision making problem based on IVNSs by applying the Inclusion Measure. For that, consider the pattern recognition problem which consists of a given set of known patterns and a given unknown pattern (sample). Our aim is to calculate the inclusion measure between each of the patterns and the sample. The maximum value shows that the sample belongs to the pattern. This process is called pattern recognition.

\section{Algorithm}

1. Consider the known set of patterns $M_{i}(i=1$ to $n)$.

2. Find the unknown sample (decision's positive ideal solution).

3. Calculate distance measure.

4. Calculate inclusion measure.

5. Choose the maximum value as the optimal decision. 


\section{Example}

Let us take the given set of three known patterns be $A, B$ and $C$ which correspond to three decision alternatives $D_{1}, D_{2}$ and $D_{3}$ respectively. Here we denote the patterns by IVNSs as $X=\left\{x_{1}, x_{2}, x_{3}\right\}$

$$
\begin{aligned}
A & =\left\{\left\langle x_{1},[0.3,0.6],[0.2,0.3],[0.3,0.4]\right\rangle,\left\langle x_{2},[0.5,0.6],[0.2,0.3],[0.3,0.4]\right\rangle,\left\langle x_{3},[0.4,0.5],[0.2,0.4],[0.7,0.9]\right\rangle\right\} \\
B & =\left\{\left\langle x_{1},[0.4,0.5],[0.2,0.3],[0.3,0.4]\right\rangle,\left\langle x_{2},[0.4,0.6],[0.1,0.3],[0.2,0.4]\right\rangle,\left\langle x_{3},[0.7,0.9],[0.2,0.3],[0.4,0.5]\right\rangle\right\} \\
C & =\left\{\left\langle x_{1},[0.6,0.7],[0.1,0.2],[0.2,0.3]\right\rangle,\left\langle x_{2},[0.6,0.7],[0.1,0.2],[0.1,0.3]\right\rangle,\left\langle x_{3},[0.3,0.6],[0.3,0.5],[0.8,0.9]\right\rangle\right\}
\end{aligned}
$$

Let the unknown sample be the positive ideal solution of decision.

Now we find the positive ideal solution by using the formula,

$$
\begin{gathered}
S^{*}=\left\{\left\langle x_{i}, \max T\left(x_{i}\right), \min I\left(x_{i}\right), \min F\left(x_{i}\right)\right\rangle, \forall i\right\} \\
S^{*}=\left\{\left\langle x_{1},[0.6,0.7],[0.1,0.2],[0.2,0.3]\right\rangle,\left\langle x_{2},[0.6,0.7],[0.1,0.2],[0.1,0.3]\right\rangle,\left\langle x_{3},[0.7,0.9],[0.2,0.3],[0.4,0.5]\right\rangle\right\}
\end{gathered}
$$

Our main purpose is to classify the pattern $S^{*}$ to one of the decision alternatives $D_{1}, D_{2}$ and $D_{3}$.

The formula for Inclusion Measure based on distance measure is $I_{d}(A, B)=1-d(A, A \cap B)$.

For that, first we calculate the intersection operation by using the formula

$$
\begin{aligned}
A \cap B=\{\langle x, & {\left[\min \left\{T_{A}^{l}(x), T_{B}^{l}(x)\right\}, \min \left\{T_{A}^{u}(x), T_{B}^{u}(x)\right\}\right], } \\
& {\left[\max \left\{I_{A}^{l}(x), I_{B}^{l}(x)\right\}, \max \left\{I_{A}^{u}(x), I_{B}^{u}(x)\right\}\right], } \\
& {\left.\left.\left[\max \left\{F_{A}^{l}(x), F_{B}^{l}(x)\right\}, \max \left\{F_{A}^{u}(x), F_{B}^{u}(x)\right\}\right]\right\rangle: x \in X\right\} }
\end{aligned}
$$

Then we get,

$$
\begin{aligned}
S^{*} & \cap A \\
& =\left\{\left\langle x_{1},[0.3,0.6],[0.2,0.3],[0.3,0.4]\right\rangle,\left\langle x_{2},[0.5,0.6],[0.2,0.3],[0.3,0.4]\right\rangle,\left\langle x_{3},[0.4,0.5],[0.2,0.4],[0.7,0.9]\right\rangle\right\} \\
& =A
\end{aligned}
$$

Similarly $S^{*} \cap B=B$ and $S^{*} \cap C=C$. 
Now, based on some distance measure, we calculate the Inclusion Measure for IVNSs as follows:

\section{(1) Hamming Distance}

$$
\begin{aligned}
& d(A, B)=\frac{1}{6} \sum_{i=1}^{n}\left[\begin{array}{l}
\left.\left|T_{A}^{l}\left(x_{i}\right)-T_{B}^{l}\left(x_{i}\right)\right|+\left|T_{A}^{u}\left(x_{i}\right)-T_{B}^{u}\left(x_{i}\right)\right|+\left|I_{A}^{l}\left(x_{i}\right)-I_{B}^{l}\left(x_{i}\right)\right|+\right] \\
\left|I_{A}^{u}\left(x_{i}\right)-I_{B}^{u}\left(x_{i}\right)\right|+\left|F_{A}^{l}\left(x_{i}\right)-F_{B}^{l}\left(x_{i}\right)\right|+\left|F_{A}^{u}\left(x_{i}\right)-F_{B}^{u}\left(x_{i}\right)\right|
\end{array}\right] \\
& d\left(S^{*}, S^{*} \cap A\right)=\frac{1}{6}\{0.3+0.1+0.1+0.1+0.1+0.1+0.1+0.1+0.1+0.1+0.2+0.1+0.3+0.4+0+0.1+0.3+0.4\} \\
& d\left(S^{*}, S^{*} \cap A\right)=\frac{1}{6}(3)=0.5 \\
& d\left(S^{*}, S^{*} \cap B\right)=\frac{1}{6}\{0.2+0.2+0.1+0.1+0.1+0.1+0.2+0.1+0+0.1+0.1+0.1+0\} \\
& d\left(S^{*}, S^{*} \cap B\right)=\frac{1}{6}(1.4)=0.23333 \\
& d\left(S^{*}, S^{*} \cap C\right)=\frac{1}{6}\{0+0.4+0.3+0.1+0.2+0.4+0.4\} \\
& d\left(S^{*}, S^{*} \cap C\right)=\frac{1}{6}(1.8)=0.3 \\
& I\left(S^{*}, A\right)=1-0.5=0.5, I\left(S^{*}, B\right)=1-0.23333=0.7666, I\left(S^{*}, C\right)=1-0.3=0.7
\end{aligned}
$$

(2) Normalized Hamming Distance

$$
\begin{gathered}
d(A, B)=\frac{1}{6 n} \sum_{i=1}^{n}\left[\begin{array}{l}
\left|T_{A}^{l}\left(x_{i}\right)-T_{B}^{l}\left(x_{i}\right)\right|+\left|T_{A}^{u}\left(x_{i}\right)-T_{B}^{u}\left(x_{i}\right)\right|+\left|I_{A}^{l}\left(x_{i}\right)-I_{B}^{l}\left(x_{i}\right)\right|+ \\
I_{A}^{u}\left(x_{i}\right)-I_{B}^{u}\left(x_{i}\right)|+| F_{A}^{l}\left(x_{i}\right)-F_{B}^{l}\left(x_{i}\right)|+| F_{A}^{u}\left(x_{i}\right)-F_{B}^{u}\left(x_{i}\right) \mid
\end{array}\right] \\
d\left(S^{*}, S^{*} \cap A\right)=\frac{1}{6(3)}(3)=0.16666, d\left(S^{*}, S^{*} \cap B\right)=\frac{1}{6(3)}(1.4)=0.07777 \\
d\left(S^{*}, S^{*} \cap C\right)=\frac{1}{6(3)}(1.8)=0.1 \\
I\left(S^{*}, A\right)=1-0.16666=0.8334, I\left(S^{*}, B\right)=1-0.07777=0.9222 \\
I\left(S^{*}, C\right)=1-0.1=0.9
\end{gathered}
$$


(3) Euclidean Distance

$$
\begin{aligned}
& d(A, B)=\left\{\frac{1}{6} \sum_{i=1}^{n}\left[\begin{array}{l}
\left|T_{A}^{l}\left(x_{i}\right)-T_{B}^{l}\left(x_{i}\right)\right|^{2}+\left|T_{A}^{u}\left(x_{i}\right)-T_{B}^{u}\left(x_{i}\right)\right|^{2}+\left|I_{A}^{l}\left(x_{i}\right)-I_{B}^{l}\left(x_{i}\right)\right|^{2}+ \\
\left|I_{A}^{u}\left(x_{i}\right)-I_{B}^{u}\left(x_{i}\right)\right|^{2}+\left|F_{A}^{l}\left(x_{i}\right)-F_{B}^{l}\left(x_{i}\right)\right|^{2}+\left|F_{A}^{u}\left(x_{i}\right)-F_{B}^{u}\left(x_{i}\right)\right|^{2}
\end{array}\right]\right\}^{\frac{1}{2}} \\
& d\left(S^{*}, S^{*} \cap A\right)=\left\{\frac{1}{6}\left[\begin{array}{l}
(0.3)^{2}+(0.1)^{2}+(0.1)^{2}+(0.1)^{2}+(0.1)^{2}+(0.1)^{2}+(0.1)^{2}+(0.1)^{2}+(0.1)^{2}+ \\
(0.1)^{2}+(0.2)^{2}+(0.1)^{2}+(0.3)^{2}+(0.4)^{2}+0+(0.1)^{2}+(0.3)^{2}+(0.4)^{2}
\end{array}\right]\right\}^{\frac{1}{2}} \\
& d\left(S^{*}, S^{*} \cap A\right)=\sqrt{\frac{1}{6}(0.74)}=\sqrt{0.12333}=0.35118 \\
& d\left(S^{*}, S^{*} \cap B\right)=\left\{\frac{1}{6}\left[\begin{array}{l}
(0.2)^{2}+(0.2)^{2}+(0.1)^{2}+(0.1)^{2}+(0.1)^{2}+(0.1)^{2}+ \\
(0.2)^{2}+(0.1)^{2}+0+(0.1)^{2}+(0.1)^{2}+(0.1)^{2}+0
\end{array}\right]\right\}^{\frac{1}{2}} \\
& d\left(S^{*}, S^{*} \cap B\right)=\sqrt{\frac{1}{6}(0.2)}=\sqrt{0.033333}=0.18257 \\
& d\left(S^{*}, S^{*} \cap C\right)=\left\{\frac{1}{6}\left[0+(0.4)^{2}+(0.3)^{2}+(0.1)^{2}+(0.2)^{2}+(0.4)^{2}+(0.4)^{2}\right]\right\}^{\frac{1}{2}} \\
& d\left(S^{*}, S^{*} \cap C\right)=\sqrt{\frac{1}{6}(0.62)}=\sqrt{0.103333}=0.32145 \\
& I\left(S^{*}, A\right)=1-0.35118=0.64882, I\left(S^{*}, B\right)=1-0.18257=0.81743 \\
& I\left(S^{*}, C\right)=1-0.32145=0.67855
\end{aligned}
$$

\section{(4) Normalized Euclidean Distance}

$$
\begin{aligned}
& d(A, B)=\left\{\frac{1}{6 n} \sum_{i=1}^{n}\left[\begin{array}{l}
\left|T_{A}^{l}\left(x_{i}\right)-T_{B}^{l}\left(x_{i}\right)\right|^{2}+\left|T_{A}^{u}\left(x_{i}\right)-T_{B}^{u}\left(x_{i}\right)\right|^{2}+\left|I_{A}^{l}\left(x_{i}\right)-I_{B}^{l}\left(x_{i}\right)\right|^{2}+ \\
\left|I_{A}^{u}\left(x_{i}\right)-I_{B}^{u}\left(x_{i}\right)\right|^{2}+\left|F_{A}^{l}\left(x_{i}\right)-F_{B}^{l}\left(x_{i}\right)\right|^{2}+\left|F_{A}^{u}\left(x_{i}\right)-F_{B}^{u}\left(x_{i}\right)\right|^{2}
\end{array}\right]\right\}^{\frac{1}{2}} \\
& d\left(S^{*}, S^{*} \cap A\right)=\sqrt{\frac{1}{6(3)}(0.74)}=\sqrt{0.041111}=0.20275
\end{aligned}
$$




$$
\begin{aligned}
& d\left(S^{*}, S^{*} \cap B\right)=\sqrt{\frac{1}{6(3)}(0.2)}=\sqrt{0.011111}=0.10540 \\
& d\left(S^{*}, S^{*} \cap C\right)=\sqrt{\frac{1}{6(3)}(0.62)}=\sqrt{0.034444}=0.18559 \\
& I\left(S^{*}, A\right)=1-0.20275=0.79725, I\left(S^{*}, B\right)=1-0.10540=0.8946 \\
& I\left(S^{*}, C\right)=1-0.18559=0.81441
\end{aligned}
$$

(5) Hausdroff Distance

$$
\begin{aligned}
d(A, B)= & \sum_{i=1}^{n} \max \left[\begin{array}{l}
\left.\left|T_{A}^{l}\left(x_{i}\right)-T_{B}^{l}\left(x_{i}\right)\right|,\left|T_{A}^{u}\left(x_{i}\right)-T_{B}^{u}\left(x_{i}\right)\right|,\left|I_{A}^{l}\left(x_{i}\right)-I_{B}^{l}\left(x_{i}\right)\right|,\right] \\
\left|I_{A}^{u}\left(x_{i}\right)-I_{B}^{u}\left(x_{i}\right)\right|,\left|F_{A}^{l}\left(x_{i}\right)-F_{B}^{l}\left(x_{i}\right)\right|,\left|F_{A}^{u}\left(x_{i}\right)-F_{B}^{u}\left(x_{i}\right)\right|
\end{array}\right] \\
d\left(S^{*}, S^{*} \cap A\right)= & \max \{0.3,0.1,0.1,0.1,0.1,0.1\}+\max \{0.1,0.1,0.1,0.1,0.2,0.1\}+ \\
& \max \{0.3,0.4,0,0.1,0.3,0.4\} \\
= & 0.3+0.2+0.4=0.9 \\
d\left(S^{*}, S^{*} \cap B\right)= & \max \{0.2,0.2,0.1,0.1,0.1,0.1\}+\max \{0.2,0.1,0,0.1,0.1,0.1\}+0 \\
= & 0.2+0.2+0=0.4 \\
d\left(S^{*}, S^{*} \cap C\right)= & 0+0+\max \{0.4,0.3,0.1,0.2,0.4,0.4\}=0.4 \\
I\left(S^{*}, A\right)= & 1-0.9=0.1, I\left(S^{*}, B\right)=1-0.4=0.6, I\left(S^{*}, C\right)=1-0.4=0.6 .
\end{aligned}
$$

(6) Normalized Hausdroff Distance

$$
\begin{aligned}
& d(A, B)=\frac{1}{n} \sum_{i=1}^{n} \max \left[\begin{array}{l}
\left|T_{A}^{l}\left(x_{i}\right)-T_{B}^{l}\left(x_{i}\right)\right|,\left|T_{A}^{u}\left(x_{i}\right)-T_{B}^{u}\left(x_{i}\right)\right|,\left|I_{A}^{l}\left(x_{i}\right)-I_{B}^{l}\left(x_{i}\right)\right|, \\
\left|I_{A}^{u}\left(x_{i}\right)-I_{B}^{u}\left(x_{i}\right)\right|,\left|F_{A}^{l}\left(x_{i}\right)-F_{B}^{l}\left(x_{i}\right)\right|,\left|F_{A}^{u}\left(x_{i}\right)-F_{B}^{u}\left(x_{i}\right)\right|
\end{array}\right] \\
& d\left(S^{*}, S^{*} \cap A\right)=\frac{1}{3}(0.9)=0.3 \\
& d\left(S^{*}, S^{*} \cap B\right)=\frac{1}{3}(0.4)=0.13333
\end{aligned}
$$




$$
\begin{aligned}
& d\left(S^{*}, S^{*} \cap C\right)=\frac{1}{3}(0.4)=0.13333 \\
& I\left(S^{*}, A\right)=1-0.3=0.7, \quad I\left(S^{*}, B\right)=1-0.13333=0.86667 \\
& I\left(S^{*}, C\right)=1-0.13333=0.86667
\end{aligned}
$$

\section{(7) Weighted Hamming Distance}

$$
d(A, B)=\frac{1}{6} \sum_{i=1}^{n} w_{i}\left[\begin{array}{l}
\left|T_{A}^{l}\left(x_{i}\right)-T_{B}^{l}\left(x_{i}\right)\right|+\left|T_{A}^{u}\left(x_{i}\right)-T_{B}^{u}\left(x_{i}\right)\right|+\left|I_{A}^{l}\left(x_{i}\right)-I_{B}^{l}\left(x_{i}\right)\right|+ \\
\left|I_{A}^{u}\left(x_{i}\right)-I_{B}^{u}\left(x_{i}\right)\right|+\left|F_{A}^{l}\left(x_{i}\right)-F_{B}^{l}\left(x_{i}\right)\right|+\left|F_{A}^{u}\left(x_{i}\right)-F_{B}^{u}\left(x_{i}\right)\right|
\end{array}\right]
$$

Here, the weight $w_{i} \in[0,1]$ and $\sum_{i=1}^{n} w_{i}=1$. Let us take $w=(0.2,0.3,0.5)$.

$$
\begin{aligned}
d\left(S^{*}, S^{*} \cap A\right)= & \frac{1}{6}\left\{\begin{array}{r}
0.2(0.3+0.1+0.1+0.1+0.1+0.1)+0.3(0.1+0.1+0.1+0.1+0.2+0.1)+ \\
0.5(0.3+0.4+0+0.1+0.3+0.4)
\end{array}\right\} \\
& =\frac{1}{6}[0.2(0.8)+0.3(0.7)+0.5(1.5)]=\frac{1}{6}(1.12)=0.186666 \\
d\left(S^{*}, S^{*} \cap B\right) & =\frac{1}{6}\{0.2(0.2+0.2+0.1+0.1+0.1+0.1)+0.3(0.2+0.1+0+0.1+0.1+0.1)+0.5(0)\} \\
& =\frac{1}{6}[0.2(0.8)+0.3(0.6)+0]=\frac{1}{6}(0.34)=0.056666 \\
d\left(S^{*}, S^{*} \cap C\right)= & \frac{1}{6}\{0.2(0)+0.3(0)+0.5(0.4+0.3+0.1+0.2+0.4+0.4)\} \\
& =\frac{1}{6}[0+0+0.5(1.8)]=\frac{1}{6}(0.9)=0.15 \\
I\left(S^{*}, A\right) & =1-0.186666=0.81333, I\left(S^{*}, B\right)=1-0.056666=0.94333, \\
I\left(S^{*}, C\right) & =1-0.15=0.85
\end{aligned}
$$

(8) Weighted Normalized Hamming Distance

$$
d(A, B)=\frac{1}{6 n} \sum_{i=1}^{n} w_{i}\left[\begin{array}{l}
\left|T_{A}^{l}\left(x_{i}\right)-T_{B}^{l}\left(x_{i}\right)\right|+\left|T_{A}^{u}\left(x_{i}\right)-T_{B}^{u}\left(x_{i}\right)\right|+\left|I_{A}^{l}\left(x_{i}\right)-I_{B}^{l}\left(x_{i}\right)\right|+ \\
\left|I_{A}^{u}\left(x_{i}\right)-I_{B}^{u}\left(x_{i}\right)\right|+\left|F_{A}^{l}\left(x_{i}\right)-F_{B}^{l}\left(x_{i}\right)\right|+\left|F_{A}^{u}\left(x_{i}\right)-F_{B}^{u}\left(x_{i}\right)\right|
\end{array}\right]
$$




$$
\begin{aligned}
& d\left(S^{*}, S^{*} \cap A\right)=\frac{1}{6(3)}(1.12)=0.062222 \\
& d\left(S^{*}, S^{*} \cap B\right)=\frac{1}{6(3)}(0.34)=0.018888 \\
& d\left(S^{*}, S^{*} \cap C\right)=\frac{1}{6(3)}(0.9)=0.05 \\
& I\left(S^{*}, A\right)=1-0.062222=0.93777, I\left(S^{*}, B\right)=1-0.018888=0.98111, \\
& I\left(S^{*}, C\right)=1-0.05=0.95
\end{aligned}
$$

\section{(9) Weighted Euclidean Distance}

$$
\begin{aligned}
& d(A, B)=\left\{\frac{1}{6} \sum_{i=1}^{n} w_{i}\left[\begin{array}{l}
\left|T_{A}^{l}\left(x_{i}\right)-T_{B}^{l}\left(x_{i}\right)\right|^{2}+\left|T_{A}^{u}\left(x_{i}\right)-T_{B}^{u}\left(x_{i}\right)\right|^{2}+\left|I_{A}^{l}\left(x_{i}\right)-I_{B}^{l}\left(x_{i}\right)\right|^{2}+ \\
\left|I_{A}^{u}\left(x_{i}\right)-I_{B}^{u}\left(x_{i}\right)\right|^{2}+\left|F_{A}^{l}\left(x_{i}\right)-F_{B}^{l}\left(x_{i}\right)\right|^{2}+\left|F_{A}^{u}\left(x_{i}\right)-F_{B}^{u}\left(x_{i}\right)\right|^{2}
\end{array}\right]\right\}^{\frac{1}{2}} \\
& d\left(S^{*}, S^{*} \cap A\right)=\left\{\frac{1}{6}[0.2(0.14)+0.3(0.09)+0.5(0.51)]\right\}^{\frac{1}{2}} \\
& =\left\{\frac{1}{6}(0.028+0.027+0.255)\right\}^{\frac{1}{2}}=\sqrt{\frac{1}{6}(0.31)}=\sqrt{0.0516666}=0.22730 \\
& d\left(S^{*}, S^{*} \cap B\right)=\left\{\frac{1}{6}[0.2(0.12)+0.3(0.08)+0.5(0)]\right\}^{\frac{1}{2}} \\
& =\left\{\frac{1}{6}(0.024+0.024+0)\right\}^{\frac{1}{2}}=\sqrt{\frac{1}{6}(0.048)}=\sqrt{0.008}=0.08944 \\
& d\left(S^{*}, S^{*} \cap C\right)=\left\{\frac{1}{6}[0.2(0)+0.3(0)+0.5(0.62)]\right\}^{\frac{1}{2}}=\sqrt{\frac{1}{6}(0.31)}=\sqrt{0.0516666}=0.22730 \\
& I\left(S^{*}, A\right)=1-0.22730=0.7727, I\left(S^{*}, B\right)=1-0.08944=0.91056 \text {, } \\
& I\left(S^{*}, C\right)=1-0.22730=0.7727
\end{aligned}
$$


(10) Weighted Normalized Euclidean Distance

$$
\begin{aligned}
& d(A, B)=\left\{\frac{1}{6 n} \sum_{i=1}^{n} w_{i}\left[\begin{array}{l}
\left.\left|T_{A}^{l}\left(x_{i}\right)-T_{B}^{l}\left(x_{i}\right)\right|^{2}+\left|T_{A}^{u}\left(x_{i}\right)-T_{B}^{u}\left(x_{i}\right)\right|^{2}+\left|I_{A}^{l}\left(x_{i}\right)-I_{B}^{l}\left(x_{i}\right)\right|^{2}+\right] I_{A}^{u}\left(x_{i}\right)-\left.I_{B}^{u}\left(x_{i}\right)\right|^{2}+\left|F_{A}^{l}\left(x_{i}\right)-F_{B}^{l}\left(x_{i}\right)\right|^{2}+\left|F_{A}^{u}\left(x_{i}\right)-F_{B}^{u}\left(x_{i}\right)\right|^{2}
\end{array}\right]\right\}^{\frac{1}{6}}=\sqrt{0.017222}=0.13123 \\
& d\left(S^{*}, S^{*} \cap A\right)=\sqrt{\frac{1}{6(3)}(0.31)}=\sqrt{\frac{1}{6(3)}(0.048)}=\sqrt{0.002666}=0.05163 \\
& d\left(S^{*}, S^{*} \cap B\right)=\sqrt{\frac{1}{6(3)}(0.31)}=\sqrt{0.017222}=0.13123 \\
& d\left(S^{*}, S^{*} \cap C\right)=\sqrt{I\left(S^{*}, A\right)}=1-0.13123=0.86877, I\left(S^{*}, B\right)=1-0.05163=0.94837 \\
& I\left(S^{*}, C\right)=1-0.13123=0.86877
\end{aligned}
$$

(11) Weighted Hausdroff Distance

$$
\begin{gathered}
d(A, B)=\sum_{i=1}^{n} w_{i} \max \left[\begin{array}{l}
\left.\left|T_{A}^{l}\left(x_{i}\right)-T_{B}^{l}\left(x_{i}\right)\right|,\left|T_{A}^{u}\left(x_{i}\right)-T_{B}^{u}\left(x_{i}\right)\right|,\left|I_{A}^{l}\left(x_{i}\right)-I_{B}^{l}\left(x_{i}\right)\right|,\right] \\
\left|I_{A}^{u}\left(x_{i}\right)-I_{B}^{u}\left(x_{i}\right)\right|,\left|F_{A}^{l}\left(x_{i}\right)-F_{B}^{l}\left(x_{i}\right)\right|,\left|F_{A}^{u}\left(x_{i}\right)-F_{B}^{u}\left(x_{i}\right)\right|
\end{array}\right] \\
d\left(S^{*}, S^{*} \cap A\right)=0.2(0.3)+0.3(0.2)+0.5(0.4)=0.06+0.06+0.2=0.32 \\
d\left(S^{*}, S^{*} \cap B\right)=0.2(0.2)+0.3(0.2)+0.5(0)=0.04+0.06+0=0.1 \\
d\left(S^{*}, S^{*} \cap C\right)=0.2(0)+0.3(0)+0.5(0.4)=0.2 \\
I\left(S^{*}, A\right)=1-0.32=0.68, I\left(S^{*}, B\right)=1-0.1=0.9, \quad I\left(S^{*}, C\right)=1-0.2=0.8 .
\end{gathered}
$$

(12) Weighted Normalized Hausdroff Distance

$$
\begin{gathered}
d(A, B)=\frac{1}{n} \sum_{i=1}^{n} w_{i} \max \left[\begin{array}{l}
\left|T_{A}^{l}\left(x_{i}\right)-T_{B}^{l}\left(x_{i}\right)\right|,\left|T_{A}^{u}\left(x_{i}\right)-T_{B}^{u}\left(x_{i}\right)\right|,\left|I_{A}^{l}\left(x_{i}\right)-I_{B}^{l}\left(x_{i}\right)\right|, \\
\left|I_{A}^{u}\left(x_{i}\right)-I_{B}^{u}\left(x_{i}\right)\right|,\left|F_{A}^{l}\left(x_{i}\right)-F_{B}^{l}\left(x_{i}\right)\right|,\left|F_{A}^{u}\left(x_{i}\right)-F_{B}^{u}\left(x_{i}\right)\right|
\end{array}\right] \\
d\left(S^{*}, S^{*} \cap A\right)=\frac{1}{3}(0.32)=0.106666
\end{gathered}
$$




$$
\begin{aligned}
d\left(S^{*}, S^{*} \cap B\right) & =\frac{1}{3}(0.1)=0.033333 \\
d\left(S^{*}, S^{*} \cap C\right) & =\frac{1}{3}(0.2)=0.066666 \\
I\left(S^{*}, A\right) & =1-0.10666=0.89334, I\left(S^{*}, B\right)=1-0.03333=0.96667, \\
I\left(S^{*}, C\right) & =1-0.06666=0.93334
\end{aligned}
$$

\section{(13) Euclidean Hausdroff Distance}

$$
\begin{aligned}
& d(A, B)=\left\{\sum_{i=1}^{n} \max \left[\begin{array}{l}
\left|T_{A}^{l}\left(x_{i}\right)-T_{B}^{l}\left(x_{i}\right)\right|^{2},\left|T_{A}^{u}\left(x_{i}\right)-T_{B}^{u}\left(x_{i}\right)\right|^{2},\left|I_{A}^{l}\left(x_{i}\right)-I_{B}^{l}\left(x_{i}\right)\right|^{2}, \\
\left|I_{A}^{u}\left(x_{i}\right)-I_{B}^{u}\left(x_{i}\right)\right|^{2},\left|F_{A}^{l}\left(x_{i}\right)-F_{B}^{l}\left(x_{i}\right)\right|^{2},\left|F_{A}^{u}\left(x_{i}\right)-F_{B}^{u}\left(x_{i}\right)\right|^{2}
\end{array}\right]\right\}^{\frac{1}{2}} \\
& d\left(S^{*}, S^{*} \cap A\right)=\{0.09+0.04+0.16\}^{\frac{1}{2}}=\sqrt{0.29}=0.538516 \\
& d\left(S^{*}, S^{*} \cap B\right)=\{0.04+0.04+0\}^{\frac{1}{2}}=\sqrt{0.08}=0.282842 \\
& d\left(S^{*}, S^{*} \cap C\right)=\sqrt{0.16}=0.4 \\
& I\left(S^{*}, A\right)=1-0.538516=0.461484, I\left(S^{*}, B\right)=1-0.282842=0.717158 \\
& I\left(S^{*}, C\right)=1-0.4=0.6
\end{aligned}
$$

\section{(14) Weighted Euclidean Hausdroff Distance}

$$
\begin{gathered}
d(A, B)=\left\{\sum_{i=1}^{n} w_{i} \max \left[\begin{array}{l}
\left.\left|T_{A}^{l}\left(x_{i}\right)-T_{B}^{l}\left(x_{i}\right)\right|^{2},\left|T_{A}^{u}\left(x_{i}\right)-T_{B}^{u}\left(x_{i}\right)\right|^{2},\left|I_{A}^{l}\left(x_{i}\right)-I_{B}^{l}\left(x_{i}\right)\right|^{2},\right]\left.\right|^{\frac{1}{2}} \\
\left|I_{A}^{u}\left(x_{i}\right)-I_{B}^{u}\left(x_{i}\right)\right|^{2},\left|F_{A}^{l}\left(x_{i}\right)-F_{B}^{l}\left(x_{i}\right)\right|^{2},\left|F_{A}^{u}\left(x_{i}\right)-F_{B}^{u}\left(x_{i}\right)\right|^{2}
\end{array}\right]\right\} \\
d\left(S^{*}, S^{*} \cap A\right)=\{0.2(0.09)+0.3(0.04)+0.5(0.16)\}^{\frac{1}{2}}=\sqrt{0.018+0.012+0.08}=\sqrt{0.11}=0.33166 \\
d\left(S^{*}, S^{*} \cap B\right)=\{0.2(0.04)+0.3(0.04)+0.5(0)\}^{\frac{1}{2}}=\sqrt{0.008+0.012}=\sqrt{0.02}=0.141421 \\
d\left(S^{*}, S^{*} \cap C\right)=\{0.2(0)+0.3(0)+0.5(0.16)\}^{\frac{1}{2}}=\sqrt{0.08}=0.282842
\end{gathered}
$$




$$
\begin{aligned}
& I\left(S^{*}, A\right)=1-0.33166=0.66834, I\left(S^{*}, B\right)=1-0.141421=0.858579 \\
& I\left(S^{*}, C\right)=1-0.282842=0.717158
\end{aligned}
$$

(15) Normalized Euclidean Hausdroff Distance

$$
\begin{gathered}
d(A, B)=\left\{\frac{1}{n} \sum_{i=1}^{n} \max \left[\begin{array}{l}
\left.\left|T_{A}^{l}\left(x_{i}\right)-T_{B}^{l}\left(x_{i}\right)\right|^{2},\left|T_{A}^{u}\left(x_{i}\right)-T_{B}^{u}\left(x_{i}\right)\right|^{2},\left|I_{A}^{l}\left(x_{i}\right)-I_{B}^{l}\left(x_{i}\right)\right|^{2},\right] I_{A}^{u}\left(x_{i}\right)-\left.I_{B}^{u}\left(x_{i}\right)\right|^{2},\left|F_{A}^{l}\left(x_{i}\right)-F_{B}^{l}\left(x_{i}\right)\right|^{2},\left|F_{A}^{u}\left(x_{i}\right)-F_{B}^{u}\left(x_{i}\right)\right|^{2}
\end{array}\right]\right\}^{\frac{1}{2}}=0.31091 \\
d\left(S^{*}, S^{*} \cap A\right)=\sqrt{\frac{1}{3}(0.29)}=\sqrt{0.096666}=0.16329 \\
d\left(S^{*}, S^{*} \cap B\right)=\sqrt{\frac{1}{3}(0.08)}=\sqrt{0.026666}=0.23093 \\
d\left(S^{*}, S^{*} \cap C\right)=\sqrt{\frac{1}{3}(0.16)}=\sqrt{0.053333}=0.23631, \\
I\left(S^{*}, A\right)=1-0.31091=0.68909, I\left(S^{*}, B\right)=1-0.16329=0.83671 \\
I\left(S^{*}, C\right)=1-0.23093=0.76907
\end{gathered}
$$

(16) Normalized Weighted Euclidean Hausdroff Distance

$$
\begin{aligned}
& d(A, B)=\left\{\frac{1}{n} \sum_{i=1}^{n} w_{i} \max \left[\begin{array}{l}
\left|T_{A}^{l}\left(x_{i}\right)-T_{B}^{l}\left(x_{i}\right)\right|^{2},\left|T_{A}^{u}\left(x_{i}\right)-T_{B}^{u}\left(x_{i}\right)\right|^{2},\left|I_{A}^{l}\left(x_{i}\right)-I_{B}^{l}\left(x_{i}\right)\right|^{2}, \\
\left|I_{A}^{u}\left(x_{i}\right)-I_{B}^{u}\left(x_{i}\right)\right|^{2},\left|F_{A}^{l}\left(x_{i}\right)-F_{B}^{l}\left(x_{i}\right)\right|^{2},\left|F_{A}^{u}\left(x_{i}\right)-F_{B}^{u}\left(x_{i}\right)\right|^{2}
\end{array}\right]\right\}^{\frac{1}{2}} \\
& d\left(S^{*}, S^{*} \cap A\right)=\sqrt{\frac{1}{3}(0.11)}=\sqrt{0.036666}=0.19148 \\
& d\left(S^{*}, S^{*} \cap B\right)=\sqrt{\frac{1}{3}(0.02)}=\sqrt{0.006666}=0.08164 \\
& d\left(S^{*}, S^{*} \cap C\right)=\sqrt{\frac{1}{3}(0.08)}=\sqrt{0.026666}=0.16329 \\
& I\left(S^{*}, A\right)=1-0.19148=0.80852, I\left(S^{*}, B\right)=1-0.08164=0.91836 \\
& I\left(S^{*}, C\right)=1-0.16329=0.83671
\end{aligned}
$$


Since $I\left(S^{*}, B\right)$ is maximum, the pattern $S^{*}$ must be classified to $B$ according to the principle of Inclusion Measure between IVNSs. Clearly, $D_{2}$ is the optimal alternative. That is, $D_{2}$ is the closest alternative to positive ideal solution.

\section{Conclusion}

We presented the Inclusion Measure concept based on some distance measures between IVNSs in a simple manner by illustrating a multi attribute decision making problem in pattern recognition method. We hope that the concept said in this paper will help the persons to upgrade the future work on Inclusion Measure to do general framework for the real practical life applications.

\section{References}

(1) F. Smarandache, Neutrosophic Set: A generalization of the Intuitionistic fuzzy set, International Journal of Pure and Applied Mathematics 24 (2005), 287-297.

(2) K. T. Atanassov, Intuitionistic Fuzzy Sets, Fuzzy Sets Syst. 20 (1) (1986) 87-96.

(3) L. A. Zadeh, Fuzzy Sets, Information and Control 8 (1965) 338-353.

(4) R. Şahin and A. Küçük, Subsethood measure for single valued neutrosophic sets, Journal of Intelligent and Fuzzy Systems, 2014, DOI: 10.3233/IFS-141304.

(5) Ridvan Şahin and Mesut Karabacak, A MADM method based on inclusion measure for IVNSs, IJEAS, ISSN: 2394-3661, Vol.2, Issue-2, Feb 2015.

(6) Sudip Bhattacharyya, Bikas Koli Roy, Pinaki Majumdar, On Distances and Similarity Measures between Two Interval Neutrosophic Sets, Journal of New Theory, ISSN: 2149-1402, 20 (2018), 27-47.

(7) V. Young, Fuzzy Subsethood, Fuzzy Sets Syst. 77 (3) (1996) 371-384. 